\title{
Mismatch repair hierarchy of $P$ seudomonas putida revealed by mutagenic ssDNA recombineering of the pyrF gene
}

\author{
by \\ Tomas Aparicioํㅜㄹ Akos Nyerges², István Nagy33,4, Csaba Pal2, \\ Esteban Martínez-García ${ }^{\star 1}$ and Víctor de Lorenzo ${ }^{1 *}$
}

\begin{abstract}
${ }^{1}$ Systems and Synthetic Biology Program, Centro Nacional de Biotecnología (CNB-CSIC), Campus de Cantoblanco, Madrid 28049, Spain 2Synthetic and Systems Biology Unit, Institute of Biochemistry and ${ }^{3}$ Sequencing Platform, Biological Research Centre, Hungarian Academy of Sciences, H-6726 Szeged, 4Sequencing Laboratory, SeqOmics Biotechnology Ltd., 6782 Mórahalom, Hungary
\end{abstract}

Running title: The MMR system of Pseudomonas putida

Keywords: Pseudomonas, recombineering, mutagenesis, mutS, mutL, mismatch repair

Originality-Significance Statement. Single-stranded DNA (sSDNA) recombineering has emerged in recent years as one of the most powerful technologies of genome editing in $E$. coli and other Enterobacteria. However, the efforts to expand the concept and the methods towards environment microorganisms such as Pseudomonas putida have been limited thus far by several gaps in our fundamental knowledge of how nucleotide mismatch repair (MMR) operates in such non-model species. One critical bottleneck is the hierarchy of recognition of different types of base mispairings as well as the need of setting up strategies for counteracting MMR and thus enabling tolerance to all types of changes. The work presented here tackles both issues and makes $P$. putida amenable to sophisticated genetic manipulations that were impossible before.

This article has been accepted for publication and undergone full peer review but has not been through the copyediting, typesetting, pagination and proofreading process which may lead to differences between this version and the Version of Record. Please cite this article as doi: $10.1111 / 1462-2920.14814$ 
* Correspondence to: Víctor de Lorenzo and Esteban Martínez-García, Centro Nacional de Biotecnología-CSIC, Campus de Cantoblanco, Madrid 28049, Spain. Tel: $34-9158545$ 36; Fax: 34-91 58545 06. E-mails: vdlorenzo@cnb.csic.es, emartinez@cnb.csic.es 
SUMMARY

The mismatch repair (MMR) system is one of the key molecular devices that prokaryotic cells have for ensuring fidelity of DNA replication. While the canonical MMR of $E$. coli involves 3 proteins (encoded by mutS, mutL and mutH), the soil bacterium Pseudomonads putida has only 2 bona fide homologues (mutS and mutL) and the sensitivity of this abridged system to different types of mismatches is unknown. In this background, sensitivity to MMR of this bacterium was inspected through single stranded (ss) DNA recombineering of the pyrF gene (the prokaryotic equivalent to yeast's URA3) with mutagenic oligos representative of every possible mispairing under either wild-type conditions, permanent deletion of mutS or transient loss of mutL activity (brought about by the thermoinducible dominant negative allele mutLE36K). Analysis of single nucleotide mutations borne by clones resistant to fluoroorotic acid (5FOA, the target of wild type PyrF) pinpointed prohibited and tolerated singlenucleotide replacements and exposed a clear grading of mismatch recognition. The resulting data unequivocally established the hierarchy $A: G<C: C<G: A<C: A, A: A, G: G, T: T, T: G, A: C, C: T<G: T, T: C$ as the one prevalent in Pseudomonas putida. This information was vital for enabling recombineering strategies aimed at single-nucleotide changes in this biotechnologically important species.

\section{INTRODUCTION}

Mutations in DNA are often caused by small insertion-deletion loops generated by strand slippage during replication and/or misincorporation of bases-by themselves or damaged by oxidative stress or other modifications (Wyrzykowski and Volkert, 2003; Putnam, 2016). The resulting base pairing mismatches are most frequently fixed by mechanisms that are remarkably conserved through the prokaryotic realm (Putnam, 2016). The steps involved in such a repair involve the recognition of an unusual structure in the DNA helix caused by the mismatch, excision of the last-synthesized strand to the site of mispairing and de novo synthesis of the earlier excised strand. In order to avoid inheritance of mutations, the critical feature of mismatch repair (MMR) systems is distinguishing between the old, non- 
modified DNA strand that acts as template and the new DNA sequence bearing the lesion. In the case where the issue has been examined in more depth (Escherichia coli), such a discrimination seems to be feasible owing to the interplay between its MMR and the dam methylation system for d(GATC) sites. According to the current model, the MMR machinery is recruited towards the strand that is transiently unmethylated after replication. This intricate process is effected through the concerted action of three proteins encoded by mutS (mispair recognition), mutL (methylated strand discrimination and recruitment of MutH) and mutH (nicking the unmethylated DNA strand), the action of which is then followed by DNA excision and resynthesis involving additional proteins like the UvrD helicase and exonucleases (Matson and Robertson, 2006).

Inspection of homologous genes in a variety of bacterial branches suggests many species-specific adaptations around the archetypal MMR of E. coli. MutS and MutL variants have been found in virtually all Gammaproteobacteria, but MutH is often missing in many other members of the group (Putnam, 2016). This raises questions on whether the same deformations of the DNA helix are recognized by the MMR systems (mostly by MutS) in all species and how DNA strand discrimination occurs in bacteria lacking muth. This question has a direct consequence on the hierarchy of mismatch recognition, as each of the 12 possible mispairings (A:G, C:C, G:A, C:A, A:A, G:G, T:T, T:G, A:C, C:T, G:T and T:C) should generate a distinct type of distortion in the DNA structure. Intuitively, the mechanism just described would predict that the bulkier the mismatch is, the easier it is to detect by the MMR system and thus fixed. But it is also possible that MutS specializes in different mispairings in diverse species. As a consequence, the MMR may become blind to some nucleotide changes, which could thus be propagated into the progeny in some hosts while others would be instead quickly removed. This originates a hierarchy of mismatch recognition by MMR, which has been clearly established in $E$. coli and other enterobacteria (Kramer et al., 1984; Babic et al., 1996; Joshi and Rao, 2001; Nyerges et al., 2016) but it less known in most others (Long et al., 2014; Long et al., 2018). Data on such recognition order is of essence for planning recombineering experiments aimed at introducing single nucleotide changes at specific genomic sites, as they can be counteracted with various efficiencies by the native 
MMR system of the target species and strains (Wang et al., 2009; Aparicio et al., 2016; Nyerges et al., 2016).

As is the case with other Pseudomonas, $P$. putida strain KT2440 has mutS and mutL, but lacks both mutH and a dam methylation system. Unlike E. coli, strand discrimination in this species could occur not through methylation but possibly through a device somehow embedded in the replication machinery itself, but the state of affairs in Pseudomonas is uncertain at this time (Oliver et al., 2002; Saumaa et al., 2006; Tark et al., 2008). In view of the growing importance of $P$. putida as a platform for synthetic biology-guided metabolic engineering and the benefits of implementing high efficiency genome editing methods (i.e. MAGE; Wang et al., 2009) and DIvERGE (Nyerges et al., 2018), it became of essence to set unequivocally the recognition preference of its native MMR system for each of the possible single nucleotide mispairs.

In the work presented below we have capitalized on the availability of a $P$. putida-born, Erf-like recombinase (called Rec2) and a simple single-stranded (ss) DNA recombineering protocol (Ricaurte et al., 2018) for exploring the whole recognition landscape of mismatches that can be introduced in the genomic DNA of strain EM42 of this species (Martinez-Garcia et al., 2014). By inspecting the distribution of single-nucleotide changes covering the whole spectrum of mispairs we authenticated the ease of replacement of given bases by any of the others in MMR-plus and MMR-minus genetic backgrounds. The outcome turned out to be similar, but not identical, to what is known for $E$. coli. The robust recombineering approach for inspecting the MMR adopted in this work has been instrumental for setting a much improved method that can be of general value for unraveling the same question-and expanding recombineering in general- to many other bacterial species.

\section{RESULTS AND DISCUSSION}

A genetic platform for inspecting MMR in P. putida 
The starting point of this work is the notion that inhibiting MMR should result in the bias-free incorporation of all possible base substitutions in the DNA helix in vivo (Nyerges, 2016). Such an inhibition could be made permanent e.g. through deletion of mutS, or transient e.g. through conditional expression of a dominant negative allele of either mutS (Wu and Marinus, 1994) or mutL (Aronshtam and Marinus, 1996; Nyerges et al., 2016). While deleting mutS is straightforward with genetic methods available for $P$. putida (see Experimental procedures), the second scenario (temporary suppression of MMR genes) required a different strategy. Inspection of the mutL (PP_4896) of $P$. putida indicated a 44 $\%$ aa identity with the orthologue of $E$. coli, which was more pronounced in their N-terminal half of the corresponding proteins-where the segments important for their function lay (Ban and Yang, 1998; Putnam, 2016). A E32K change in such an N-terminal of $E$. coli's MutL is known to generate a mutated, inactive protein that, when over expressed from a plasmid, behaves as a negative dominant allele capable of impairing the activity of the MMR machinery of $E$. coli in the presence of the chromosomal, wild-type copy (Aronshtam and Marinus, 1996; Nyerges et al., 2016). This location is equivalent to conserved amino acid position 36 of the P. putida's homologue (Fig. 1, Supplementary Fig. S1) and therefore we reasoned that overexpression in vivo of the variant mut $_{\mathrm{E} 36 \mathrm{~K}} \mathrm{PP}$ could bring about the same effect in this species. Finally, in order to enter mismatches of different types in a target DNA sequence, we thought of exploiting the ability of the Rec2 recombinase to enable the invasion of the replication fork of $P$. putida by synthetic single-stranded (ss) oligonucleotides in vivo (Ricaurte et al., 2018).

On these bases, we generated, in one hand, an MMR-null strain by erasing mutS altogether (Table 1), which was used as a reference for complete elimination of mismatch repair. P. putida EM42 $\triangle$ mutS was constructed by deleting a $0.7 \mathrm{~Kb}$ region of the gene PP_1626 by ssDNA recombineering/CRISPR-Cas9 (see details in Experimental Procedures). The phenotype of this strain was tested with a rifampicin resistance $\left(\mathrm{RifR}^{\mathrm{R}}\right)$ assay, which was performed as a proxy of the mutational state caused by the deletion. Results shown in Supplementary Fig. S2A accredited that-as expected- the deleted mutant underwent a much higher spontaneous mutational regime than its parental strain, which can be attributed to the loss of MMR system. 
On the other hand, we constructed two conditional expression plasmids for either rec2 alone or the same but assembled in the same transcriptional unit together with mut $\mathrm{E}_{\mathrm{E} 3 \mathrm{~K}} \mathrm{PP}^{\mathrm{PP}}$. In either case, the expression cargo (whether rec2 alone or rec2-mutL $\mathrm{E} 36 \mathrm{~K}^{\mathrm{PP}}$ ) was placed under the control of the heatinducible cl857-PL system of vector pSEVA2514 (Aparicio et al., 2019b), which allows intense expression of the genes inserted downstream after a short induction. The results of these operations were plasmids pSEVA2514-rec2 (GenBank No MN180223) and pSEVA2514-rec2-mutL ${ }_{E 36 K}{ }^{\text {PP }}$ (GenBank No MN180222; Fig. 1). Supplementary Fig. S2B shows that this expression system overperformed the previous recombineering platform based on induction of the recombinase with 3-methyl-benzoate (3MB) through the xylS-Pm device (Ricaurte et al., 2018), with an order of magnitude of improvement in editing efficiency. Simultaneous expression of the recombinase and mut $\mathrm{E}_{\mathrm{E} 6 \mathrm{~K}}{ }^{\mathrm{PP}}$ with this system should therefore enable the survival of mismatches generated by recombineering within a given time window, which would otherwise be removed by an active MMR system (see below). In order to optimize the recombineering protocol, different induction times for the thermal induction of rec2 were tested (Supplementary Fig. S2C), as little as 5 min being sufficient for achieving high levels of allelic replacements in the standard recombineering assay described by (Ricaurte et al., 2018).

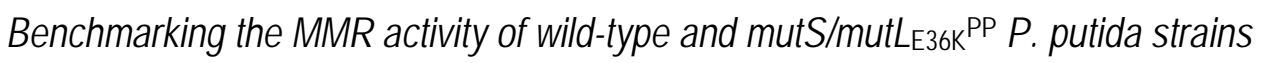

In order to obtain some reference values on the ability of MutL $\mathrm{E} 36 \mathrm{~K}^{\mathrm{PP}}$ to allow inheritance of mismatches in P. putida, we designed two recombineering oligonucleotides (SR and NR, Fig. 2A, Table 1). These enter single-nucleotide changes that-using the $E$. coli system as an orientation-represent the extremes of the ability of the MMR system to remove mismatches. But at the same time they cause easily detectable phenotypes if incorporated in the replication fork. In one case (SR oligonucleotide; (Ricaurte et al., 2018)) the sequence was designed for targeting the rpsL gene (PP_0449) of $P$. putida EM42. This gene encodes the 30S ribosomal protein S12 and a change in the wild-type codon AAA $(\mathrm{K} 43) \rightarrow$ ACA (T43) confers streptomycin resistance $\left(\mathrm{Sm}^{\mathrm{R}}\right)$. Upon Rec2-mediated recombineering, SR should generate an A:G mismatch predicted to show low sensitivity to the MMR system (Babic et al., 1996; Nyerges et al., 2016). If maintained, the change enters in the $r p s L$ the mutation $A \rightarrow C$ conferring 
$\mathrm{Sm}^{\mathrm{R}}$. By the same token, oligonucleotide NR (Table 1) was designed to target the gene gyrA (PP_1767) of $P$. putida, (encoding a DNA gyrase subunit) for executing an amino acid change D87N known to confer resistance to nalidixic acid $(\mathrm{NaI})$ in $E$. coli and $P$. aeruginosa (Yoshida et al., 1990; Kureishi et al., 1994). The mismatch introduced by NR causes the same change in the gyrA gene of $P$. putida $(\mathrm{GAC})$ D87 $\rightarrow($ AAT) N87 but in this case making two modifications at once $(G \rightarrow A$ and $C \rightarrow T)$. Both G:T and C:A mismatches thus ought to survive the action of MMR to result in resistance to nalidixic acid $(\mathrm{Nal})^{\mathrm{R}}$. NR thus had a considerable diagnostic value, as G:T and C:A mismatches are highly sensitive to the MMR system (using again E. coli as provisional reference; Nyerges et al., 2016).

For benchmarking the experimental system to investigate the mispairing preferences of the MMR system of $P$. putida, strain EM42 was separately transformed with pSEVA2514-rec2 and with pSEVA2514-rec2-mutL ${ }_{E 36 K}{ }^{\mathrm{PP}}$. Also $P$. putida EM42 $\Delta$ mutS was transformed with pSEVA2514-rec2. The resulting transformants were expected to support SSDNA recombineering of the mutagenic oligonucleotides described above upon thermal activation of the $P_{L}$ promoter. However, they are anticipated to have MMR in a different operational state: $P$. putida $\Delta$ mutS (pSEVA2514-rec2) has a permanently disabled system due to the deletion of the main component of MMR machinery (mutS); $P$. putida EM42 (pSEVA2514-rec2) has a wild-type MMR system; and P. putida EM42 (pSEVA2514-rec2mut $_{\mathrm{E} 36 \mathrm{~K}^{\mathrm{PP}}}$ ) has a functional wild-type MMR system at $30{ }^{\circ} \mathrm{C}$ which can be transiently inactivated upon thermal induction and overexpression of the dominant-negative mutL $\mathrm{E} 36 \mathrm{~K}^{\mathrm{PP}}$ allele.

The results of the recombineering experiments run with these 3 strains upon electroporation of the SR and NR oligonucleotides are shown in Fig. 2B. In one hand, SR incorporation to the replication fork yielded $S m^{R}$ cells through a single nucleotide change in which the involved mismatch $(A: G)$ is expected to be poorly recognized and thus left unrepaired in cells bearing an intact MMR machinery. On the other hand, recombineering of the NR oligonucleotide should generate NalR cells but the G:T and C:A mismatches could be readily recognized and fixed by MMR. The expected outcome of these experiments in the wild type background of $P$. putida EM42 (pSEVA2514-rec2) should thus show much higher recombineering efficiency with SR than with NR. In contrast, when the MMR system is 
impaired—whether permanently in strain $P$. putida $\Delta$ mutS (pSEVA2514-rec2) or transiently in P. putida EM42 (pSEVA2514-rec2-mut $L_{E 36 K}{ }^{P P}$ ) the frequencies of allelic replacements using SR and NR should converge.

These predictions were not only confirmed by the data of Fig. $2 \mathrm{~B}$, but the results also allowed quantification of the recombineering efficiencies under the various conditions. Specifically, Fig. 2B revealed a difference of two orders of magnitude between $\mathrm{Sm}^{\mathrm{R}}$ and $\mathrm{Nal}$ clones resulting from the recombineering experiments with the wild-type strain P. putida EM42 (pSEVA2514-rec2) and SR/NR oligos, respectively. In contrast, the frequencies of $\mathrm{Nal}^{\mathrm{R}}$ resistant clones in $\mathrm{P}$. putida $\Delta$ mutS (pSEVA2514-rec2) upon transformation with the NR oligonucleotide increased to the levels of the SmR clones of the same strain treated with the SR oligo. These data confirmed that MMR is altogether eliminated in the $\Delta$ mutS strain and that this lesion abolishes any bias in mispair recognition and repairing process. Finally, when the MutLE36K ${ }^{\mathrm{PP}}$ protein was overexpressed in strain P. putida EM42 (pSEVA2514-rec2-mut $L_{E 36 K}{ }^{P P}$ ) the frequencies of $S m^{R}$ and NalR resulting respectively from treatments with the SR and NR oligos were very similar. Taken together, the results of Fig. 2 showed that the inherent activity of the MMR native of $P$. putida clearly discriminates different types of mismatches (in the case tested: low activity against $A: G$ and high activity against $G: T+C: A)$ which can be transiently and effectively silenced in vivo upon expression of the dominant negative allele E36K of mutL. On this basis we set out to explore the whole landscape of single-nucleotide mispairings allowed or not in $P$. putida, as explained next.

Rationale for unraveling the hierarchy of the MMR system of $\mathrm{P}$. putida

In order to characterize the bias in the detection/repair of single nucleotide changes by the MMR system of $P$. putida, a set of four mutagenic oligonucleotides targeting the pyrF gene (PP_1815) of $P$. putida were designed. This gene, which is equivalent to yeast's URA3, encodes orotidine 5'-phosphate decarboxylase and its inactivation makes cells to become resistant to fluoroorotic acid (5FOA ; (Galvao and de Lorenzo, 2005). The oligos for mutagenic recombineering of pyrF share the same sequence 
within the gene but bear four distinct positions fully degenerated (Supplementary Table S1, Fig. 3A), targeting nucleotides A (oligo PYR_A), C (oligo PYR_C), T (oligo PYR_T) and G (oligo PYR_G). The oligonucleotides encode also in all cases a $C \rightarrow$ A change which turns GAA codon E58 into TAA (Stop) in the midst of the pyrF ORF. When incorporated into the chromosome, this change thus generates cells with a truncated, non-functional pyrF gene, which become then uracil auxotrophs and 5FOAR. This set of oligonucleotides can therefore generate all possible mismatches in vivo during a ssDNA recombineering experiment, thereby exposing them to the endogenous MMR activity-whether fully active, fully inactive or transiently inhibited. While all mutants that have incorporated the oligos in the genome can be selected by growing the cells in the presence of uracil and 5FOA, the frequency of the accompanying changes can be quantified by PCR and deep sequencing of the targeted region of pyrF.

Nucleotide mispairing preferences of the MMR system of $\mathrm{P}$. putida EM42

On the basis of the above, the predisposition of $P$. putida MMR system to recognize and repair different DNA mismatches and the ability of the MutL $\mathrm{E} 36 \mathrm{~K}^{\mathrm{PP}}$ protein to effectively abrogate the bias was inspected. To this end, the oligos employed in the ssDNA recombineering experiments bear mismatches with the chromosomal DNA that are incorporated into the replication fork by the action of the Rec2 recombinase. Since the endogenous MMR activity can repair the mismatches to various degrees, the frequency of 5FOAR mutants become a quantitative assay of MMR activity. Recombineering experiments were first performed with equimolar mixtures of PYR_A/T/C/G oligonucleotides in the wild-type, $M \mathrm{MR}^{+}$ background of strain P. putida EM42 (pSEVA2514-rec2) as explained in Experimental procedures. Note that the objective of this experiment was not assessing recombineering efficiencies but describing the hierarchy of the MMR system of $P$. putida in the strains under study. Yet, recombineering frequencies with PYR_A/T/C/G oligos were clearly higher in the MMR-defective strains i.e. P. putida $\Delta$ mutS (pSEVA2514-rec2) and P. putida EM42 (pSEVA2514-rec2-mut $L_{\mathrm{E} 36 \mathrm{~K}}{ }^{\mathrm{PP}}$ ), than in the wild-type strain $P$. putida EM42 (pSEVA2514-rec2) at least by one order of magnitude. Therefore, the data shown below do not reflect actual recombineering performance but normalised percentages of mismatch repair in the three genetic backgrounds under study. After selection on M9-Citrate-Ura-5FOA plates, 500 colonies 
were reisolated in the same medium, then pooled together and genomic DNA extracted. This DNA pool was used as the template for amplification of pyrF gene by PCR and the resulting amplicons were analyzed by Illumina deep sequencing (see Experimental Procedures for details). Fig. 3B shows the relative frequency of the allelic replacements observed in this experiment, reflecting the bias of the wildtype $P$. putida EM42 MMR system to detect and repair single nucleotide mispairings. The results allowed us to establish the following hierarchy of mismatch recognition from less to more sensitive (and thus more to less permissive to changes) : $A: G<C: C<G: A<C: A, A: A, G: G, T: T, T: G, A: C, C: T<G: T$, T:C. This grading is comparable, but not identical, to that found in E. coli (Kramer et al., 1984; Nyerges et al., 2016). Similarly to this species of reference, the MMR system of $P$. putida EM42 shows very low sensitivity to A:G and C:C mismatches (Babic et al., 1996; Nyerges et al., 2016). However, the T:T mismatch is poorly recognized/repaired in P. putida while the MMR system of $E$. coli has a much higher sensitivity to it. On the other hand, G:T and T:C mismatches have remarkably high repair rates in $P$. putida EM42 (i.e., less permissive), while $E$. coli do not show such noticeable differences. It is also noteworthy that identical mismatches are repaired differently depending on the specific position of the targeted nucleotide. While A:G ( mediated by PYR_A) shows $39.7 \%$ of allelic replacement, G:A (mediated by PYR_G) accounts only for 19.8 \%. Even more dissimilar figures are found in the mismatches C:T and T:C (mediated by PYR_C and PYR_T) with replacement frequencies of 0.4 and $0.02 \%$, respectively. The same happens with $\mathrm{C}: \mathrm{A} / \mathrm{A}: \mathrm{C}$ and $\mathrm{T}: \mathrm{G} / \mathrm{G}: \mathrm{T}$ (see Fig. S3). This can be explained by the mismatch sequence context: the two bases adjacent to the mispaired position have been reported to influence the repair activity of $E$. coli, with differential rates as high as 50 -fold between heterogeneous contexts (Modrich, 1991; Foster et al., 2018). All in all, the MMR discrimination encompasses three orders of magnitude i.e. from A:G (39.7\% efficiency) vs. to T:C (0.02\%). According to these data, C:A/G:T, the mismatches involved in the NalR phenotype mediated by NR oligonucleotide (Fig. 2B) should be highly sensitive to MMR, but in fact the difference with the upper extreme is only two logs-surely due to the presence of the second C:A mismatch in the NR oligo. This can be explained in light of (Sawitzke et al., 2011), namely, if two mismatches become simultaneously incorporated, the MMR recognizes them differently from the individual mutations, a phenomenon that seems to occur also in P. putida. Note also that MMR action on the mismatches occurs always in the 
newly synthesized strand that incorporates the mutagenic oligonucleotide. As commented in the Introduction above, this hints towards a mechanism of template/newly synthesized DNA discrimination in $P$. putida which cannot depend on dam methylation, an open question that deserves further studies.

To clarify the role of MMR in the recognition/repair just described, the same recombineering experiments were performed with strains $P$. putida $\Delta$ mutS (pSEVA2514-rec2) and $P$. putida EM42 (pSEVA2514-rec2-mut $L_{E 36 K}{ }^{P P}$ ) and the results were plotted along with those with the wild-type stain (Fig. 4A). A heatmap with the mean values of the resulting allelic replacements is shown in Fig. 4B. Both using of the MMR defective strain and heat induction of the mut $L_{\mathrm{E} 36 \mathrm{~K}^{\mathrm{PP}}}$ allele resulted in remarkable reduction of the recognition/repair bias of $P$. putida MMR system (Supplementary Fig. S3 shows detailed information of the allelic replacement frequencies obtained). However, inspection of the actual figures of 5FOAR mutants indicated that a complete loss of MMR largely equalizes, but does not completely abolish, the bias towards stable inheritance of mutations generated by mispairings. Yet, the differential rate of repair in the $\mathrm{MMR}^{+}$strain between high and low sensitive mismatches is close to 1000 -fold, while the permanent or transient removal of MMR reduces the distance to not more than 4fold (see Supplementary Fig. S3 for details). The rates of allelic replacements in mismatches very sensitive to MMR repair are particularly important in strain P. putida EM42 (pSEVA2514-rec2mut $_{\mathrm{E} 36 \mathrm{~K}^{\mathrm{PP}}}$ ) as they increase to $7 \%$ in the case of G:T (compared to a mere $0.04 \%$ in the wild-type host) and to $5 \%$ for $\mathrm{T}: \mathrm{C}\left(0.02 \%\right.$ in the $\mathrm{MMR}^{+}$strain). These data not only sheds light on the recognition preference and fixing of nucleotide mismatches in $P$. putida but also accredit the dominant negative activity of MutL $\mathrm{E}_{6 \mathrm{~K}} \mathrm{PP}$ in this species and suggest general method for momentarily supressing MMR in bacteria subjected to a recombineering protocol.

Transient expression of mut $L_{E 36 K} P P$ inhibits MMR but does not cause whole-genome mutagenesis

As indicated in Supplementary Fig. S2A, the loss of mutS multiplies the spontaneous mutagenesis rate of $P$. putida by at least 2 orders of magnitude as revealed with a simple count of Rif ${ }^{R}$ mutants in the population. One remaining question regarding the recombineering results above was whether the same 
could be brought about by the transient thermoinduction of mut $\mathrm{E}_{\mathrm{E} 6 \mathrm{~K}}{ }^{\mathrm{PP}}$ during the period of time involved in the recombineering experiments. This piece of information is of essence for judging whether plasmid pSEVA2514-rec2-mutL ${ }_{E 36 K}{ }^{\text {PP }}$ could be a useful construct for implementing specific single-nucleotide changes in the genome of $P$. putida through Rec2-mediated recombineering without the complication of suspecting off-target, concurrent mutations. To sort this out we first adopted a RifR fluctuation-like assay for quantifying the impact of inhibiting the MMR system in the mutational rate of the strains under study. Fig. 5 shows that overexpression mut $\mathrm{E}_{\mathrm{E} 6 \mathrm{~K}}{ }^{\mathrm{PP}}$ had a minor effect on the frequency of spontaneous appearance of Rif ${ }^{R}$ clones of $P$. putida EM42 (approximately a 4-fold increase). In contrast, the $\Delta$ mutS strain under the same conditions exhibited $>100$ fold increase in mutations leading to Rif $^{\mathrm{R}}$ compared to the wild-type $P$. putida, a value in agreement with the earlier data shown in Supplementary Fig. S2A. In order to asses further the appearance of random mutations during the transient inactivation of the MMR system with mut $_{\mathrm{E} 36 \mathrm{~K}^{\mathrm{PP}}}$, two $\mathrm{Sm} \mathrm{R}^{\mathrm{R}}$ and two NalR colonies recovered from recombineering experiments of strain P. putida EM42 (pSEVA2514-rec2-mutL ${ }_{E 36 \mathrm{~K}}{ }^{\mathrm{PP}}$ ) with SR and NR oligonucleotides, respectively, were submitted to whole genome sequencing. In addition, a SmR clone originated in the treatment of the control P. putida EM42 (pSEVA2514-rec2) strain expressing only Rec2 was also analyzed. Genomic DNA of each of the 5 strains was purified and sequenced by Illumina. As shown in Supplementary Table S2, zero to three SNPs were detected in strains transiently expressing mut $_{\mathrm{E} 36 \mathrm{~K}}{ }^{\mathrm{PP}}$, while no mutations could be observed in the control $\mathrm{MMR}^{+}$strain. This demonstrated a very low level of spontaneous mutations when transiently inhibiting the MMR system of $P$. putida- low enough to consider thermoinduction of mut $\mathrm{E}_{\mathrm{E} 6 \mathrm{~K}}{ }^{\mathrm{PP}}$ an ideal asset for implementing high-efficiency genome editing methods in P. putida.

\section{Conclusion}

The results of the experiments described above provide three valuable pieces of information on how $P$. putida handles mismatches in the DNA helix of its genomic DNA caused by replication errors, misincorporation of damaged bases or (as exploited in this work) deliberate mispairings artificially introduced by means of recombineering strategies. The advantage of the last is that one can 
recapitulate all possible changes in a controlled fashion, as we have done in the present work. First, $P$. putida has an active MMR system that includes at least the mutS and mutL homologs of $E$. coli and brings about a distinct hierarchy of mismatch recognition and suppression. But possibly, the MMR system of $P$. putida (as is the case with $P$. aeruginosa (Oliver et al., 2002) comprises other activities as well: permanent or transient suppression of these genes dramatically reduce, but does not entirely eliminate the bias in tolerance to different types of mismatches. Second, as in any MMR device known in other bacteria, the system discriminates the old template strand of DNA from the newly synthesized sequence that bears the mismatched nucleotide. How this happens in the absence of dam methylation is unknown, although similarly to the case of Bacillus it could involve the beta clamp of the replication machinery (Simmons et al., 2008). And third, transient expression of the dominant negative allele E36K of mutL along with the rec2 recombinase (any possibly any other ssDNA recombinase active in this species) creates a window of opportunity for introduction of all types of chromosomal single-base changes without significant offsite mutations. This property, empowered by plasmid pSEVA2514-rec2mutL $_{\mathrm{E} 36 \mathrm{~K}} \mathrm{PP}$ (Fig. 1) will enable expansion of advanced methods of recombineering-based genome engineering such as DIvERGE (Nyerges et al., 2018) or pORTMAGE-based technology (Nyerges et al., 2016) towards this environmentally and biotechnologically important bacterium.

\section{EXPERIMENTAL PROCEDURES}

Strains, media and general procedures

Liquid LB was used as routine growth media (10 $\mathrm{g} \mathrm{l}^{-1}$ tryptone, $5 \mathrm{~g} \mathrm{l}^{-1}$ yeast extract, and $\left.5 \mathrm{~g} \mathrm{l}^{-1} \mathrm{NaCl}\right)$ for E. coli and P. putida strains used in this study (Table 1). Glycerol-free Terrific Broth (TB; $12 \mathrm{~g} \mathrm{l}^{-1}$ tryptone, $24 \mathrm{~g} \mathrm{l}^{-1}$ yeast extract, $2 \mathrm{~g} \mathrm{l}^{-1} \mathrm{KH}_{2} \mathrm{PO}_{4}, 9.4 \mathrm{~g} \mathrm{l}^{-1} \mathrm{~K}_{2} \mathrm{HPO}_{4}$ ) was used for after-electroporation recovery during recombineering experiments. Bacterial strains were cultivated with shaking $(170 \mathrm{rpm})$ at $30^{\circ} \mathrm{C}$ (P. putida) or $37^{\circ} \mathrm{C}$ (E. coli). M9 minimal media (Sambrook et al., 1989) was supplemented, when stated, with $0.2 \%$ w/v citrate for $P$. putida growth. Solid media was prepared adding $15 \mathrm{~g} / \mathrm{L}$ of agar to liquid media. When necessary, liquid and solid media were supplemented with $50 \mu \mathrm{g} \mathrm{ml}^{-1}$ of kanamycin 
(Km), $15 \mu \mathrm{g} \mathrm{ml}^{-1}$ of gentamicin (Gm), $30 \mu \mathrm{g} \mathrm{ml}^{-1}$ of chloramphenicol $(\mathrm{Cm}), 100 \mu \mathrm{g} \mathrm{ml}^{-1}$ of streptomycin (Sm), $100 \mu \mathrm{g} \mathrm{ml}^{-1}$ rifampicin (Rif), $50 \mu \mathrm{g} \mathrm{ml}^{-1}$ of nalidixic acid (Nal), $20 \mu \mathrm{g} \mathrm{ml}^{-1}$ of Uracil (Ura) or $250 \mu \mathrm{g}$ $\mathrm{ml}^{-1}$ of 5 -fluoroorotic acid (5-FOA). Standard DNA manipulations were conducted according to manufacturer recommendations and previously established protocols (Sambrook et al., 1989). Gibson Assembly was carried out as outlined in (Aparicio et al., 2017) using a home-made reaction mixture. Genomic DNA was isolated with the DNAeasy® UltraClean ${ }^{\circledR}$ Microbial Kit (Qiagen). Following manufacturer recommendations, PCR amplifications for cloning purposes were performed with Q5 polymerase (New England Biolabs) while DNA Amplitools Master Mix was used for diagnosis PCRs. Plasmids were introduced in P. putida strains via tripartite mating as described in (Martinez-Garcia and de Lorenzo, 2012). Oligonucleotides were purchased from Sigma with the exception of PYR_C, PYR_A, PYR_G, PYR_T and all PCR primers used for pyrF deep sequencing, which were synthesized at the Nucleic Acid Synthesis Laboratory of the Biological Research Centre, Hungarian Academy of Sciences, Szeged (Hungary) and purified using high-performance liquid chromatography (HPLC). Primers were suspended in 1× Tris-EDTA (TE) buffer (pH 8.0) at $100 \mu \mathrm{M}$ final concentration.

\section{Plasmid construction}

To obtain pSEVA2514-rec2, in which the rec2 recombinase is under the control of the heat-inducible cl857-P $P_{L}$ expression system, the xyIS-Pm induction module of pSEVA258-rec2 was substituted with the thermo-inducible expression module of pSEVA2514. Both plasmids were restricted with Pacl/Avrll and the $6.0 \mathrm{~Kb}$ band of pSEVA258-rec2 was ligated to the $1.4 \mathrm{~Kb}$ band of pSEVA2514 and the ligation transformed in E. coli CC118. For the construction of pSEVA2514-rec2-mutL $L_{E 36 K} P$, in which mut $L_{E 36 K}{ }^{P P}$ allele is co-expressed with rec2, the corresponding sequence was first assembled in the pSEVA258-ssr and later on transferred to pSEVA2514-rec2. pSEVA258-sSr-mutL E36K $^{P P}$ was generated as follows: first, mutL of $P$. putida KT2440 $(2.0 \mathrm{~Kb})$ was colony amplified with oligos mutL-KT-Fw/mutL-KT-Rv $(\mathrm{Tm}=65$ ${ }^{\circ} \mathrm{C}, 1$ min. elongation, Q5 polymerase). This PCR fragment was Gibson assembled with BamHI/Sphl restricted pSEVA258-SSr and the assembly mix transformed in E. coli CC118. The resulting pSEVA258ssr-mutL was used as a template for amplifying two fragments: a $0.9 \mathrm{~Kb}$ fragment containing the 3 '-end 
of ssr and the $5^{\prime}$-end of mutL with Gibson-PP-Beta-Fw/ mutLKT-Gibson-2 (Tm= $60{ }^{\circ} \mathrm{C}, 30 \mathrm{sec}$. elongation, Q5 polymerase) and a fragment containing a $0.4 \mathrm{~Kb}$ segment of mutL with mutLKT-Gibson3/ mutLKT-Gibson-4 (Tm= $71{ }^{\circ} \mathrm{C}, 30 \mathrm{sec}$. elongation, Q5 polymerase). mutLKT-Gibson-2 and mutLKTGibson-3 primers incorporate the single nucleotide change responsible of the amino acid change $\mathrm{E} 36 \rightarrow \mathrm{K} 36$ in the mutL ORF to generate mutLE36K. pSEVA258-ssr-mutL was cut with EcoRI and the 8.4 Kb fragment, containing the $5^{\prime}$-end of ssr and the $3^{\prime}$-end of mutL, was Gibson-assembled with the two PCR fragments described above to eventually generate pSEVA258-ssr-mutL ${ }_{E 36 K}{ }^{P P}$. Finally, this plasmid was used as a template for amplifying mutLE36K with primers mutLE36K-Gib-Fw/ mutLE36KGib-Rv (Tm= $65^{\circ} \mathrm{C}, 1 \mathrm{~min}$. Elongation, Q5 polymerase) and the resulting $2.0 \mathrm{~Kb}$ fragment was Gibson assembled with pSEVA2514-rec2 restricted with Xbal/HindIII. The assembly mix was transformed in $E$. coli CC118 to obtain pSEVA2514-rec2- mutLE36K PP. pSEVA231-C-mutS1 bears a CRISPR array with a spacer targeting the mutS gene of $P$. putida KT2440. Spacer design and cloning was performed as described previously (Aparicio et al., 2018, 2019a). Briefly, oligonucleotides cr-mutS-S-1 and cr-mutSAS-1 were annealed and the resulting dsDNA fragment was ligated into PSEVA231-CRISPR restricted with Bsal. Ligation was transformed in E. coli CC118. All plasmids constructed in this work, either by Gibson Assembly or by restriction/ligation, were transformed in E. coli CC118 calcium chloride competent cells, selected in LB-Km solid media and colonies checked by miniprep+restriction. Inserts were fully sequenced (Macrogen Spain) to verify the accuracy of the constructs.

Construction of P. putida EM42 $\Delta$ mutS strain by recombineering/CRISPR-Cas9

The deletion protocol described in (Aparicio et al., 2018) was applied on P. putida EM42 bearing pSEVA658-ssr and pSEVA421-Cas9tr plasmids. Recombineering with MAGE-mutS-2 oligonucleotide and CRISPR-Cas9 counterselection with pSEVA231-C-mutS1 plasmid was used to delete a $0.7 \mathrm{~Kb}$ segment of the mutS gene (PP_1626) of $P$. putida EM42. One out of fifty colonies checked by PCR with primers mutS-check $3 /$ mutS-check $4\left(\mathrm{Tm}=55^{\circ} \mathrm{C}, 30\right.$ seconds elongation) yielded the $0.6 \mathrm{~Kb}$ fragment expected for the deletion mutant. 


\section{Rifampicin Assay}

The mutational rate of $P$. putida EM42 $\Delta$ mutS and its parental strain ( $P$. putida EM42) was estimated by monitoring the appearance of rifampicin resistant $(\mathrm{Rif} R)$ colonies. Overnight cultures grown in LB were adjusted to $\mathrm{OD}_{600} \approx 1.0$ and $1 \mathrm{ml}\left(\sim 10^{9}\right.$ cells) of each sample was centrifuged 1 minute at $11.000 \mathrm{rpm}$. The pellets were re-suspended in $100 \mu \mathrm{I}$ of LB and plated on LB-Rif solid media. Rif R colonies were counted after 24 hours of incubation at $30^{\circ} \mathrm{C}$. Two independent replicas were done and the medians and standard deviations were represented as the frequencies of Rif ${ }^{R}$ mutants per $10^{9}$ cells.

ssDNA recombineering protocol mediated by thermal induction

Recombineering experiments were accomplished basically as described in (Ricaurte et al., 2018). Some modifications were implemented to trigger the activation of the thermo-inducible cl857-PL expression system of pSEVA2514 derivatives driving the expression of rec2 and mutLE36K ${ }^{\mathrm{PP}}$ genes. Overnight cultures of $P$. putida EM42 bearing the plasmids under study were back-diluted to $O D_{600}=0.1$ in a total volume of $20 \mathrm{ml}$ of LB-Km in $100 \mathrm{ml}$ Erlenmeyer flasks. Cultures were incubated at $30^{\circ} \mathrm{Cl} 170 \mathrm{rpm}$ until $\mathrm{OD}_{600}=0.4-0.5$. Then, flasks were transferred to a water bath at $42^{\circ} \mathrm{C}$ for 5 minutes with gentle shaking to increase quickly the temperature and induce the expression of Rec2/ Mut $\mathrm{E}_{\mathrm{E} 6 \mathrm{~K}}{ }^{\mathrm{PP}}$ proteins. Flasks were incubated 10 additional minutes at $42^{\circ} \mathrm{C}$ in an air shaker at $250 \mathrm{rpm}$ (15 minutes of total induction at $42^{\circ} \mathrm{C}$ ) and then cooled down in ice for 5 minutes to stop the thermal induction. When stated, different induction times were applied with shorter or longer incubations in the air shaker. Competent cells were prepared at RT by centrifuging cultures at $3220 \mathrm{~g} / 5$ minutes and washing the pellets three consecutive times with 10,5 and $1 \mathrm{ml}$ of $300 \mathrm{mM}$ sucrose solution. Pellets were finally resuspended in $200 \mu \mathrm{l}$ of the same solution. One hundred microliters of this suspension were added with $1 \mu$ of the recombineering oligonucleotide (stock at $100 \mathrm{mM}$ ), mixed thoroughly and the mixture transferred to an electroporation 2 mm-gap width cuvette (Bio-Rad). Electrotransformation was performed in a Micropulser ${ }^{\mathrm{TM}}$ device (BioRad Laboratories, Hercules, CA, USA) at $2.5 \mathrm{kV}$ and cultures were immediately inoculated in $5 \mathrm{ml}$ of fresh TB and recovered overnight at $30^{\circ} \mathrm{Cl} 170 \mathrm{rpm}$. Several dilutions of the recovered cultures were 
plated in the appropriate selective and non-selective solid media depending on the current experiment (see below).

SSDNA recombineering experiments with SR and NR oligonucleotides

Recombineering with oligonucleotides SR (A:G mismatch, low MMR sensitivity; $A \rightarrow C$ change produces a Sm-resistant phenotype) and NR (G:T and C:A mismatches, high MMR sensitivity; $G \rightarrow A$ and $C \rightarrow T$ changes produce a Nal-resistant phenotype) was performed as described in the previous section on strains P. putida EM42/ pSEVA2514-rec2 ( wild type MMR system), P. putida EM42 2 mutS/ pSEVA2514-rec2 ( inactive MMR system) and P. putida EM42/ pSEVA2514-rec2- mutL ${ }_{\mathrm{E} 36 \mathrm{~K}}{ }^{\mathrm{PP}}$ (transient MMR inactivation upon expression of mutL $\mathrm{E} 36 \mathrm{~K}^{\mathrm{PP}}$ protein). After overnight recovery, dilutions $10^{-2}, 10^{-3}$, $10^{-4}$ and $10^{-5}$ of SR and NR electroporated cultures were plated on LB-Sm and LB-Nal, respectively, to select cells harbouring the allelic replacements. To estimate the number of viable cells, dilutions $10^{-7}$ and $10^{-8}$ of were plated on LB. Plates were incubated 18 hours at $30^{\circ} \mathrm{C}$ and absolute colony counts were taken. The recombineering frequency (RF) was calculated as the ratio between the number of antibiotic-resistant colonies and the number of viable cells. This ratio was normalized to $10^{9}$ viable cells. In order to check the accuracy of the allelic replacements, ten clones from each strain/oligonucleotide experiment were PCR amplified either for rpsL gene (Sm-resistant colonies coming from SR experiments- oligos rpsL-Fw/ rpsL-Rv, $\mathrm{Tm}=57^{\circ} \mathrm{C}$, 45 seconds elongation, $0.8 \mathrm{~Kb}$ product) or for gyrA gene (Nal-resistant colonies from NR experiments-oligos gyrA-Fw/ gyrA-Rv, Tm=57 ${ }^{\circ} \mathrm{C}, 45$ seconds elongation, $0.4 \mathrm{~Kb}$ product). PCRs were purified and sequenced with rpsl-Fw and gyrA-Fw, respectively (Macrogen Spain). All 60 clones analyzed showed the expected changes introduced by the recombineering oligonucleotides without additional mutations in the region sequenced.

MMR recognition hierarchy in $P$. putida EM42

For a more detailed characterization of the MMR system of $P$. putida EM42, the three strains studied above were subjected to recombineering with a mixture of oligonucleotides PYR_A, PYR_C, PYR_T 
and PYR_G. $10 \mu \mathrm{l}$ of each oligonucleotide at $100 \mathrm{mM}$ were mixed and $2 \mu \mathrm{l}$ of the mixture $(0.5 \mu \mathrm{l}$ of each oligonucleotide) were used for electrotransformation. The SsDNA recombineering protocol mediated by thermal induction was applied as explained before but cultures were allowed to recover only 5 hours at $30^{\circ} \mathrm{C} / 170 \mathrm{rpm}$ since longer recovery times in pyrF-targeted experiments were reported to decrease the appearance of pyrF- mutants (Ricaurte et al., 2018). After recovery, several dilutions of each culture $\left(10^{-2}, 10^{-3}\right)$ were plated on M9-Citrate-Ura-5FOA and plates were incubated 48 hours at 30 ${ }^{\circ} \mathrm{C}$ to allow the slow-growing pyrF- colonies to appear. Five hundred colonies were streaked in the same media and incubated as before. The 500 streaks were pooled together by suspension in $2 \mathrm{ml}$ of water, the sample centrifuged 1 minute at 11.000 rpm and the pellets used for genomic DNA (gDNA) extraction with DNeasy ${ }^{\circledR}$ UltraClean ${ }^{\circledR}$ Microbial Kit (Qiagen). A negative control experiment was also performed with $P$. putida EM42/ pSEVA2514-rec2 electroporated without any oligonucleotide. In this case, the post-electroporated culture was recovered 5 hours and plated in M9-Citrate-Ura-5FOA (10-1 and 10-2 dilutions) and M9-Citrate (10-5 and $10^{-6}$ dilutions). As expected, only few colonies appeared on the selective media, all of them showing the fast-growing phenotype typical of spontaneous mutants, nonpyrF related, described elsewhere (Galvao and de Lorenzo, 2005; Aparicio et al., 2016). A pool of approximately 10.000 colonies rescued from the M9-Citrate plates were used for gDNA extraction and served as a control of the downstream process of deep amplicon sequencing of pyrF. An independent replica of this set of four experiments was performed and gDNA samples from both replicas were used to estimate the activity of the MMR system by deep amplicon sequencing of the pyrF targeted region.

\section{Deep amplicon sequencing of pyrF}

To determine the allelic composition of pyrF at the oligonucleotide-target site, we utilized a previously described Illumina MiSeq deep sequencing protocol (Nyerges et al., 2016). To create Illumina sequencing libraries, a 138 nucleotide-long region of pyrF in $P$. putida that was targeted by recombineering-oligonucleotides (PYR_G, PYR_A, PYR_T and PYR_C), was PCR amplified from the previously isolated, pooled gDNA samples using the corresponding barcoded primer pairs specifically designed for each experiment/sample (Supplementary Table S1). To multiplex sequencing samples on 
Illumina MiSeq, barcoded PCR primers were designed based on a previously published protocol (Kozich et al., 2013) and consisted of the appropriate Illumina adaptor sequences, a 10 nucleotide-long pad sequence, and a 2 nucleotide-long linker besides the terminal genomic target-specific primer sequences. Besides barcoded PCR primers, custom Illumina sequencing primers according to (Kozich et al., 2013) were also designed (Supplementary Table S1). Next, the pyrF oligo-target region from each gDNA sample was amplified in $4 \times 25 \mu$ l volumes, consisting of $50 \mu \mathrm{I} 2 \times$ Q5 Hot-Start MasterMix (New England Biolabs), $2 \mu \mathrm{I}$ of the corresponding sample-specific, barcoded, reverse Illumina primer $(100 \mu \mathrm{M})$ plus $2 \mu \mathrm{I}$ PYR_ILMF $(100 \mu \mathrm{M})$ primer, $200 \mathrm{ng}$ template gDNA and $45 \mu$ I nuclease-free $\mathrm{H}_{2} \mathrm{O}$. PCRs were performed in thin-wall PCR tubes in a BioRad CFX96 qPCR machine with the following thermal profile: $98^{\circ} \mathrm{C} 3$ minutes, 23 cycles of $\left(98^{\circ} \mathrm{C} 15\right.$ seconds, $62{ }^{\circ} \mathrm{C} 20$ seconds, $72{ }^{\circ} \mathrm{C} 20$ seconds) and a final extension of $72{ }^{\circ} \mathrm{C}$ for 5 minutes. Following PCRs, the 180 basepair-long amplicons were purified by using a Zymo Research DNA Clean and Concentrator ${ }^{\text {TM }}$ Kit according to the manufacturer's protocol (Zymo Research) and eluted in in $30 \mu \mathrm{I} 1 \times$ Tris-EDTA (TE) buffer (pH 8.0). To prepare samples for sequencing, amplicons were quantified using Qubit dsDNA BR assay kit (Thermo Fisher Scientific), mixed, and libraries were sequenced on an Illumina MiSeq instrument using v2 paired-end 2×250-cycle sequencing kit (Illumina). To perform sequencing, the Illumina MiSeq cartridges were supplemented with $100 \mu \mathrm{M}$ stocks of our custom Illumina sequencing primers (Supplementary Table S1). After sequencing, raw sequencing reads were de-multiplexed according to their corresponding barcodes. The average sequencing read counts were 160000 per sample. Next, the overlapping read-pairs were identified and merged to yield one template-read from each combined sequencing read using pandaseq V2.8 (Masella et al., 2012). Reads were then trimmed to an error probability threshold of 0.001 (Phred quality $=30$ ) using readtools 1.5.2 (Gomez-Sanchez and Schlotterer, 2018). Merged paired-end reads were then mapped to their corresponding reference sequence (P. putida pyrF-PP_1815) by using bowtie2 2.3.4 (Langmead) in "--very-sensitive-local" mode and the nucleotide composition was extracted for each nucleotide position within the oligo-targeted region. Allelic replacement frequencies at each oligo-targeted nucleotide positions were quantified by measuring the distribution and ratio of nucleotide substitutions for each reference nucleotide position (Nyerges et al., 2016). Finally, the allelic replacement frequency of each individual substitution was normalized to the sum of all substitutions 
detected in the experiment. Data from two independent replicas of each experimental condition were used to calculate medians and standard deviations.

Mutational rate measurement by a fluctuation-like assay

A rifampicin resistance fluctuation assay was performed with $P$. putida EM42 (pSEVA2514-rec2) $P$. putida $\Delta$ mutS (pSEVA2514-rec2) and P. putida EM42 (pSEVA2514-rec2- mutL ${ }_{\mathrm{E} 36 \mathrm{~K}}{ }^{\mathrm{PP}}$ ). The strains were inoculated in $3 \mathrm{ml}$ of LB-Km and incubated overnight at $30{ }^{\circ} \mathrm{C} / 170 \mathrm{rpm}$. In order to mimic the experimental conditions of a standard recombineering experiment, overnight cultures were back-diluted to $\mathrm{OD}_{600} \sim 0,1 \mathrm{in} 3 \mathrm{ml}$ fresh LB-Km and incubated at $30^{\circ} \mathrm{C} / 170 \mathrm{rpm}$ until $\mathrm{OD}_{600} \sim 0,5$. Cultures were then placed in a water bath at $42{ }^{\circ} \mathrm{C}$ for 5 minutes with gentle shaking, transferred to an air shaker at 42 ${ }^{\circ} \mathrm{C} / 250 \mathrm{rpm} / 10 \mathrm{~min}$ (total incubation at $42{ }^{\circ} \mathrm{C}=15 \mathrm{~min}$ ) and incubated at $4{ }^{\circ} \mathrm{C}$ for $5 \mathrm{~min}$. After overnight growth at $30^{\circ} \mathrm{C} / 170 \mathrm{rpm}$ aliquots of each culture were plated on LB and LB-Rif and plates incubated 24 hours at $30{ }^{\circ} \mathrm{C}$. Total colony count was done and the data from fifteen independent replicas of the experiment were used to calculate the mutational rate of each strain by using the Ma-Sandri-Sarkar Maximum Likelihood Estimator method and the FALCOR web tool (Hall et al., 2009).

Whole genome sequencing and bioinformatics for SNPs detection

Genomic DNA samples of $P$. putida EM42 query strains were sequenced in Macrogen Inc. (Korea). Truseq PCR Free Libraries of 350 bp were processed in Illumina Hiseq2500 (2x100 bp) flow cells (output coverage $\sim 900 \mathrm{x}$ ). Quality of raw data was analyzed using FASTQ files with FastQC tool (https://www.bioinformatics.babraham.ac.uk/projects/fastqc/). No quality issues were detected and Illumina reads were aligned to P. putida KT2440 genome (NC 002947.4) using "bwa aln" and "bwa sampe" commands with default parameters (Li and Durbin, 2010). Alignment files in SAM format were compressed, coordinate-sorted and indexed using "samtools view -bS", "samtools sort" and "samtools index" commands, respectively (Li et al., 2009). Before coordinate-sorted step, duplicated reads (paired reads aligning exactly at the same genomic coordinates, considered as PCR artifacts) were removed 
with "samtools rmdup" command. Since the genome of $P$. putida EM42 contains 10 deletions of variable size compared with the reference genome of $P$. putida KT2440, genomic regions with no coverage were detected in SAM/BAM files using "bedtools genomecov -bga" (Quinlan and Hall, 2010) and parsing the output with "grep -w 0\$". SNP detection was carried-out using "samtools mpileup -B" and "bcftools call m" (Li, 2011). Biological impact of detected polymorphisms was determined with snpEff tool setting upstream and downstream gene regions 500 bp in size (- upDownStreamLen 500;Cingolani et al., 2012). Only variants with QUAL > 200 and coverage (DP) > 200 were considered for SNP validation.

\section{ACKNOWLEDGEMENTS}

This work was funded by the HELIOS Project of the Spanish Ministry of Science BIO 2015-66960-C3-2R (MINECO/FEDER); MADONNA (H2020-FET-OPEN-RIA-2017-1-766975), BioRoboost (H2020NMBP-BIO-CSA-2018), and SYNBIO4FLAV (H2020-NMBP/0500) Contracts of the European Union and the S2017/BMD-3691 InGEMICS-CM funded by the Comunidad de Madrid (European Structural and Investment Funds). CS was supported by grants from the European Research Council H2020-ERC2014-CoG 648364, the Wellcome Trust, GINOP-2.3.2-15-2016-00020, GINOP-2.3.2-15-2016-00014 and the Lendület Program of the Hungarian Academy of Sciences. AN was supported by a PhD fellowship from the Boehringer Ingelheim Fonds. Authors declare no conflict of interest.

\section{Supplementary Information}

Figure S1. Alignment of MutL proteins of E. coli and P. putida KT2440

Figure S2. Mutational activity of $P$. putida EM42 $\Delta$ mutS strain and recombineering performance of Rec2 recombinase under the $c 18567-\mathrm{P}_{L}$ expression system

Figure S3. Allelic replacement frequencies mediated by recombineering with PYR oligonucleotides. All mismatches are expressed chromosomal: synthetic

Supplementary Table S1. Oligonucleotides used in this study 
Supplementary Table S2. Off-target mutations during transient inhibition of MMR System.

\section{REFERENCES}

Aparicio, T., de Lorenzo, V., and Martínez-García, E. (2017) Broadening the SEVA plasmid repertoire to facilitates genomic editing of Gram-negative bacteria. In Hydrocarbon and Lipid Microbiology Protocols: Genetic, Genomic and System Analyses of Pure Cultures. McGenity, T.J., Timmis, K.N., and Nogales, B. (eds). Berlin, Heidelberg: Springer Berlin Heidelberg, pp. 9-27.

Aparicio, T., de Lorenzo, V., and Martinez-Garcia, E. (2018) CRISPR/Cas9-Based counterselection boosts recombineering efficiency in Pseudomonas putida. Biotechnol J 13: e1700161.

Aparicio, T., de Lorenzo, V., and Martinez-Garcia, E. (2019a) CRISPR/Cas9-enhanced ssDNA recombineering for Pseudomonas putida. Microb Biotechnol 12: 1076-1089.

Aparicio, T., de Lorenzo, V., and Martinez-Garcia, E. (2019b) Improved thermotolerance of genomereduced Pseudomonas putida EM42 enables effective functioning of the PL /cl857 system. Biotechnol J 14: e1800483.

Aparicio, T., Jensen, S.I., Nielsen, A.T., de Lorenzo, V., and Martinez-Garcia, E. (2016) The Ssr protein (T1E_1405) from Pseudomonas putida DOT-T1E enables oligonucleotide-based recombineering in platform strain $P$. putida EM42. Biotechnol J 11: 1309-1319.

Aronshtam, A., and Marinus, M.G. (1996) Dominant negative mutator mutations in the mutL gene of Escherichia coli. Nucl Acids Res 24: 2498-2504.

Babic, I., Andrew, S.E., and Jirik, F.R. (1996) MutS interaction with mismatch and alkylated base containing DNA molecules detected by optical biosensor. Mutat Res 372: 87-96.

Ban, C., and Yang, W. (1998) Crystal structure and ATPase activity of MutL: implications for DNA repair and mutagenesis. Cell 95: 541-552.

Boyer, H.W., and Roulland-Dussoix, D. (1969) A complementation analysis of the restriction and modification of DNA in Escherichia coli. J Mol Biol 41: 459-472. 
Cingolani, P., Platts, A., Wang le, L., Coon, M., Nguyen, T., Wang, L. et al. (2012) A program for annotating and predicting the effects of single nucleotide polymorphisms, SnpEff: SNPs in the genome of Drosophila melanogaster strain w1118 iso-2; iso-3. Fly (Austin) 6: 80-92.

Foster, P.L., Niccum, B.A., Popodi, E., Townes, J.P., Lee, H., Mohammedlsmail, W., Tang, H. Determinants of base-pair substitution patterns revealed by whole-genome sequencing of DNA mismatch repair defective Escherichia coli. Genetics 209: 1029-1042.

Galvao, T.C., and de Lorenzo, V. (2005) Adaptation of the yeast URA3 selection system to Gramnegative bacteria and generation of a $\triangle$ betCDE Pseudomonas putida strain. Appl Environ Microbiol 71: 883-892.

Gomez-Sanchez, D., and Schlotterer, C. (2018) ReadTools: A universal toolkit for handling sequence data from different sequencing platforms. Mol Ecol Resour 18: 676-680.

Hall, B.M., Ma, C.X., Liang, P., and Singh, K.K. (2009) Fluctuation analysis CalculatOR: a web tool for the determination of mutation rate using Luria-Delbruck fluctuation analysis. Bioinformatics 25: 1564-1565.

Joshi, A., and Rao, B.J. (2001) MutS recognition: multiple mismatches and sequence context effects. J Biosci 26: 595-606.

Kessler, B., de Lorenzo, V., and Timmis, K.N. (1992) A general system to integrate lacZ fusions into the chromosomes of gram-negative eubacteria: regulation of the $\mathrm{Pm}$ promoter of the TOL plasmid studied with all controlling elements in monocopy. Mol Gen Genet 233: 293-301.

Kozich, J.J., Westcott, S.L., Baxter, N.T., Highlander, S.K., and Schloss, P.D. (2013) Development of a dual-index sequencing strategy and curation pipeline for analyzing amplicon sequence data on the MiSeq Illumina sequencing platform. Appl Environ Microbiol 79: 5112-5120.

Kramer, B., Kramer, W., and Fritz, H.-J. (1984) Different base/base mismatches are corrected with different efficiencies by the methyl-directed DNA mismatch-repair system of E. coli. Cell 38: 879-887. 
Kureishi, A., Diver, J.M., Beckthold, B., Schollaardt, T., and Bryan, L.E. (1994) Cloning and nucleotide sequence of Pseudomonas aeruginosa DNA gyrase gyrA gene from strain PAO1 and quinolone-resistant clinical isolates. Antimicrob Agents Chemother 38: 1944-1952.

Li, H. (2011) A statistical framework for SNP calling, mutation discovery, association mapping and population genetical parameter estimation from sequencing data. Bioinformatics 27: 2987-2993.

Li, H., and Durbin, R. (2010) Fast and accurate long-read alignment with Burrows-Wheeler transform. Bioinformatics 26: 589-595.

Li, H., Handsaker, B., Wysoker, A., Fennell, T., Ruan, J., Homer, N. et al. (2009) The Sequence Alignment/Map format and SAMtools. Bioinformatics 25: 2078-2079.

Long, H., Miller, S.F., Williams, E., and Lynch, M. (2018) Specificity of the DNA mismatch repair system (MMR) and mutagenesis bias in bacteria. Mol Biol Evol 35: 2414-2421.

Long, H., Sung, W., Miller, S.F., Ackerman, M.S., Doak, T.G., and Lynch, M. (2014) Mutation rate, spectrum, topology, and context-dependency in the DNA mismatch repair-deficient Pseudomonas fluorescens ATCC948. Genome Biol Evol 7: 262-271.

Manoil, C., and Beckwith, J. (1985) TnphoA: a transposon probe for protein export signals. Proc Natl Acad Sci USA 82: 8129-8133.

Martinez-Garcia, E., and de Lorenzo, V. (2012) Transposon-based and plasmid-based genetic tools for editing genomes of Gram-negative bacteria. Methods Mol Biol 813: 267-283.

Martinez-Garcia, E., Nikel, P.I., Aparicio, T., and de Lorenzo, V. (2014) Pseudomonas 2.0: genetic upgrading of $P$. putida KT2440 as an enhanced host for heterologous gene expression. Microb Cell Fact 13: 159.

Masella, A.P., Bartram, A.K., Truszkowski, J.M., Brown, D.G., and Neufeld, J.D. (2012) PANDAseq: paired-end assembler for illumina sequences. BMC Bioinformatics 13: 31.

Matson, S.W., and Robertson, A.B. (2006) The UvrD helicase and its modulation by the mismatch repair protein MutL. Nucl Acids Research 34: 4089-4097. 
Modrich, P. (1991) Mechanisms and biological effects of mismatch repair. Annu Rev Genet 25: 229253.

Nyerges, A., Csorgo, B., Nagy, I., Balint, B., Bihari, P., Lazar, V. et al. (2016) A highly precise and portable genome engineering method allows comparison of mutational effects across bacterial species. Proc Natl Acad Sci USA 113: 2502-2507.

Nyerges, A., Csorgo, B., Draskovits, G., Kintses, B., Szili, P., Ferenc, G. et al. (2018) Directed evolution of multiple genomic loci allows the prediction of antibiotic resistance. Proc Natl Acad Sci USA 115: E5726-E5735.

Oliver, A., Baquero, F., and Blázquez, J. (2002) The mismatch repair system (mutS, mutL and uvrD genes) in Pseudomonas aeruginosa: molecular characterization of naturally occurring mutants. Mol Microbiol 43: 1641-1650.

Putnam, C.D. (2016) Evolution of the methyl directed mismatch repair system in Escherichia coli. DNA Repair 38: 32-41.

Quinlan, A.R., and Hall, I.M. (2010) BEDTools: a flexible suite of utilities for comparing genomic features. Bioinformatics 26: 841-842.

Ricaurte, D.E., Martinez-Garcia, E., Nyerges, A., Pal, C., de Lorenzo, V., and Aparicio, T. (2018) A standardized workflow for surveying recombinases expands bacterial genome-editing capabilities. Microb Biotechnol 11: 176-188.

Sambrook, J., Fritsch, E.F., and Maniatis, T. (1989) Molecular Cloning: A Laboratory Manual. Cold Spring Harbor Press

Saumaa, S., Tarassova, K., Tark, M., Tover, A., Tegova, R., and Kivisaar, M. (2006) Involvement of DNA mismatch repair in stationary-phase mutagenesis during prolonged starvation of Pseudomonas putida. DNA Repair 5: 505-514.

Sawitzke, J.A., Costantino, N., Li, X.T., Thomason, L.C., Bubunenko, M., Court, C., and Court, D.L. (2011) Probing cellular processes with oligo-mediated recombination and using the knowledge gained to optimize recombineering. $\mathrm{J} \mathrm{Mol} \mathrm{Biol} \mathrm{407:} \mathrm{45-59.}$ 
Simmons, L.A., Davies, B.W., Grossman, A.D., and Walker, G.C. (2008) $\beta$ clamp directs localization of mismatch repair in Bacillus subtilis. Mol Cell 29: 291-301.

Tark, M., Tover, A., Koorits, L., Tegova, R., and Kivisaar, M. (2008) Dual role of NER in mutagenesis in Pseudomonas putida. DNA Repair 7: 20-30.

Wang, H.H., Isaacs, F.J., Carr, P.A., Sun, Z.Z., Xu, G., Forest, C.R., and Church, G.M. (2009) Programming cells by multiplex genome engineering and accelerated evolution. Nature 460 : 894-898.

Wu, T.H., and Marinus, M.G. (1994) Dominant negative mutator mutations in the mutS gene of Escherichia coli. J Bacteriol 176: 5393-5400.

Wyrzykowski, J., and Volkert, M.R. (2003) The Escherichia coli methyl-directed mismatch repair system repairs base pairs containing oxidative lesions. J Bacteriol 185: 1701-1704.

Yoshida, H., Bogaki, M., Nakamura, M., and Nakamura, S. (1990) Quinolone resistance-determining region in the DNA gyrase gyrA gene of Escherichia coli. Antimicrob Agents Chemother 34: 1271-1272. 
Table 1. Bacterial strains and plasmids used in this work.

\begin{tabular}{|c|c|c|}
\hline Strain or plasmid & Relevant characteristics ${ }^{a}$ & $\begin{array}{l}\text { Reference or } \\
\text { source }\end{array}$ \\
\hline \multicolumn{3}{|l|}{ Escherichia coli } \\
\hline \multirow[t]{2}{*}{ CC118 } & Cloning host; $\Delta$ (ara-leu) araD $\Delta$ lacX174 galE galK phoA & (Manoil and \\
\hline & thiE1 rpsE(SpR) rpoB (Rif $\mathrm{R}) \arg E(\mathrm{Am})$ recA1 & Beckwith, 1985) \\
\hline \multirow[t]{3}{*}{ HB101 } & Helper strain used for conjugation; $\mathrm{F}^{-} \lambda^{-} h s d S 20\left(\mathrm{rB}^{-} \mathrm{mB}^{-}\right)$ & (Boyer and \\
\hline & recA13 leuB6(Am) araC14 $\Delta$ (gpt-proA)62 lacY1 galK2(Oc) & Roulland- \\
\hline & xyl-5 mtl-1 thiE1 rpsL20(Sm) glnX44(AS) & Dussoix, 1969) \\
\hline \multicolumn{3}{|l|}{ Pseudomonas putida } \\
\hline \multirow[t]{3}{*}{ EM42 } & KT2440 derivative; $\Delta$ prophage1 $\Delta$ prophage4 $\Delta$ prophage3 & (Martinez- \\
\hline & $\Delta$ prophage $2 \quad \Delta \operatorname{Tn} 7 \quad \Delta e n d A-1 \quad \Delta$ endA-2 $\quad \Delta h s d R M S$ & Garcia et al., \\
\hline & $\Delta$ flagellum $\Delta \operatorname{Tn} 4652$ & 2014) \\
\hline EM42 $\Delta$ mutS & EM42 derivative; $\Delta$ mutS & This work \\
\hline \multicolumn{3}{|l|}{ Plasmids } \\
\hline \multirow[t]{2}{*}{ pSEVA2514 } & Inducible expression vector; oriV(RFS1010); cargo [cl857- & (Aparicio et al., \\
\hline & $\mathrm{P}_{L}$; standard multiple cloning site; $\mathrm{Km}^{\mathrm{R}}$ & 2019b) \\
\hline \multirow[t]{2}{*}{ pSEVA258-ssr } & pSEVA258 derivative bearing the ssr recombinase; oriV & (Ricaurte et al., \\
\hline & (RFS1010); cargo [xylS-Pm $\rightarrow s s r] ; \mathrm{Km}^{\mathrm{R}}$ & 2018) \\
\hline pSEVA258-sSr-mutL ${ }_{E 36 K}{ }^{P P}$ & $\begin{array}{l}\text { pSEVA258 derivative bearing the ssr recombinase and } \\
\text { mutL }_{E 36 K^{P P}} \text { allele; oriV (RFS1010); cargo }[x y / S-P m \rightarrow s s r-\end{array}$ & This work \\
\hline
\end{tabular}

This article is protected by copyright. All rights reserved. 
mutL $\left._{\mathrm{E} 36 K^{\mathrm{PP}}}\right] ; \mathrm{Km}^{\mathrm{R}}$

pSEVA2514-rec2

pSEVA2514-rec2- mut $_{\mathrm{E} 36 \mathrm{~K}}{ }^{\mathrm{PP}}$

PSEVA231-CRISPR

pSEVA231-C-mutS1

pSEVA421-Cas9tr

pSEVA658-sSr

pRK600
pSEVA2514 derivative bearing the rec2 recombinase; This work oriV(RFS1010); cargo [cl857- $\mathrm{P}_{L} \rightarrow$ rec2]; $\mathrm{Km}^{\mathrm{R}}$

pSEVA2514 derivative bearing the rec2 recombinase and This work mutt $_{\mathrm{E} 36 \mathrm{~K}}^{\mathrm{PP}}$ allele; oriV(RFS1010); cargo [c1857-PL $\rightarrow$ rec2-

mutL $\left._{\mathrm{E} 36 K^{P}}{ }^{P}\right] ; \mathrm{Km}^{\mathrm{R}}$

pSEVA231 derivative bearing the CRISPR array; oriV (Aparicio et al., (pBBR1); Km ${ }^{R}$

pSEVA231 derivative bearing the CRISPR array with a This work mutS spacer; oriV (pBBR1); Km

pSEVA421 derivative bearing the cas9 gene and tracrRNA; (Aparicio et al., oriV (RK2); $\mathrm{Sm}^{\mathrm{R}} / \mathrm{Sp}^{\mathrm{R}}$ 2018)

pSEVA658 derivative bearing the ssr recombinase; oriV (Aparicio et al., (RSF1010) ); cargo [xylS-Pm $\rightarrow s s r] ; G m^{R}$

Helper plasmid used for conjugation; oriV(ColE1), RK2 (Kessler et al., $(\mathrm{mob}+\mathrm{tra}+) ; \mathrm{Cm}^{\mathrm{R}}$ 1992)

a Antibiotic markers: Km, kanamycin; Rif, rifampicin; Sm, streptomycin; Sp, spectinomycin; Cm, chloramphenicol; Gm, gentamicin 


\section{CAPTIONS TO FIGURES}

Figure 1. Plasmids used in this study.
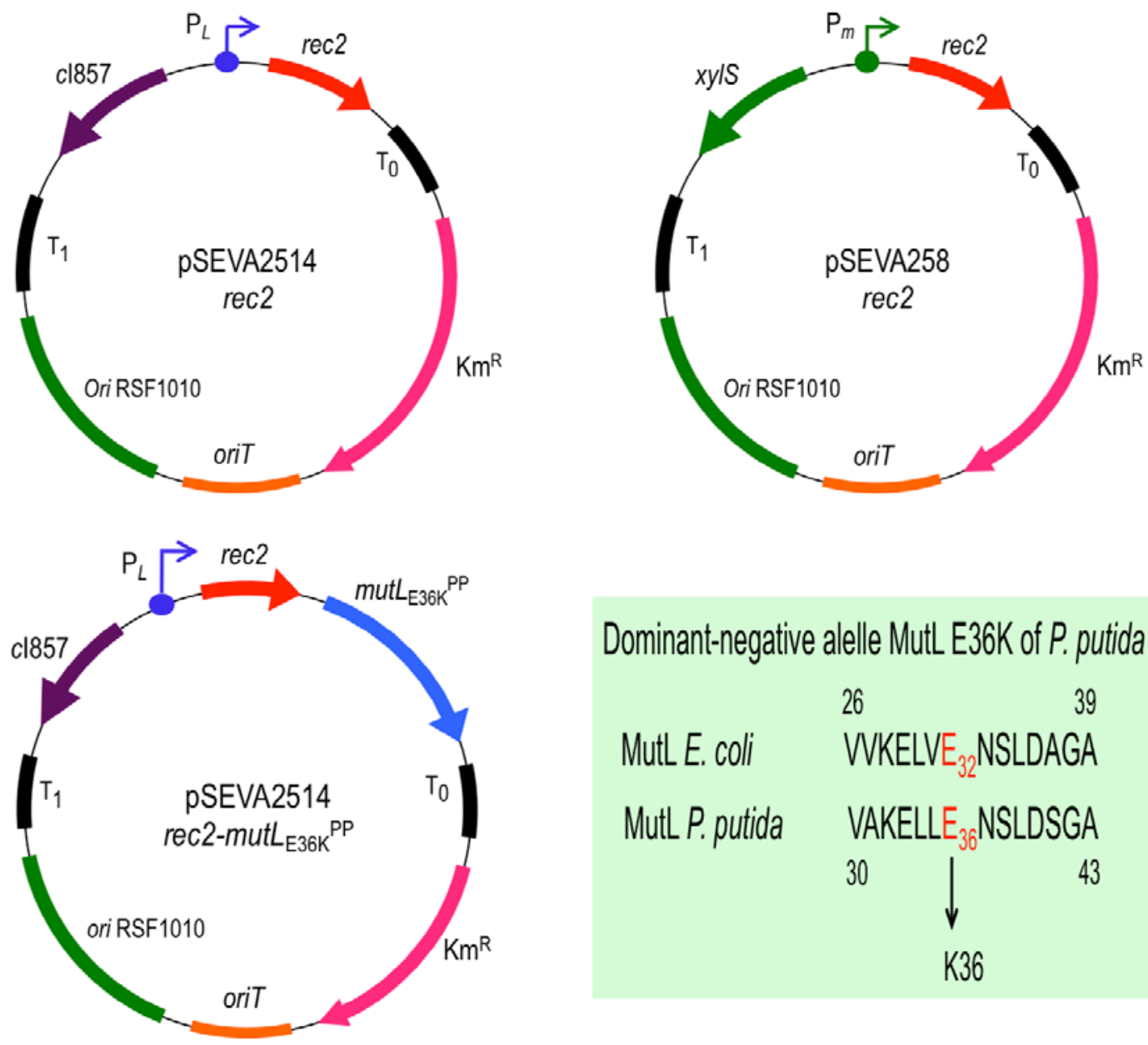

Dominant-negative alelle MutL E36K of $P$. putida

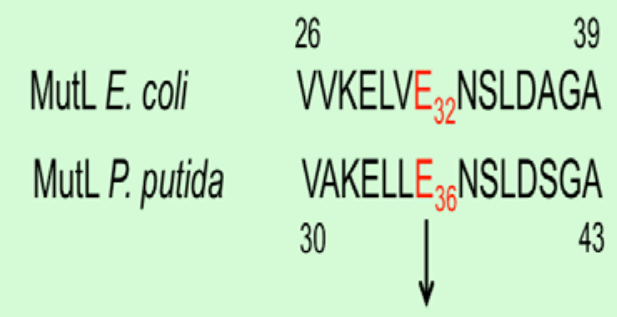

K36

Structure of plasmids promoting recombineering are shown ( $T_{0}$ and $T_{1}$, transcriptional terminators; $K m$, Kanamycin resistance gene; oriT, origin of transfer; ori RSF1010, origin of replication; cl857- $P_{L}$, temperature inducible expression system; $x y / S-\mathrm{P}_{m}$, expression system inducible by 3-MB; $r e c 2$, recombinase; mut $L_{\mathrm{E} 36 \mathrm{~K}} \mathrm{PP}$, dominant-negative allele of mutL). A conserved amino acid stretch of $E$. coli and $P$. putida KT2440 MultL proteins is also shown. The change $\mathrm{E} \rightarrow \mathrm{K}$, responsible of the dominant- 
negative phenotype over MMR system, is highlighted in red (see Supplementary Fig. S1 for complete alignment). Pictures are not drawn to scale. pSEVA2514-rec2 map derives from (Ricaurte et al., 2018). 
Figure 2. The impairment of MMR system of $P$. putida allows unbiased detection/repair of two mismatches with MMR differential sensitivity.
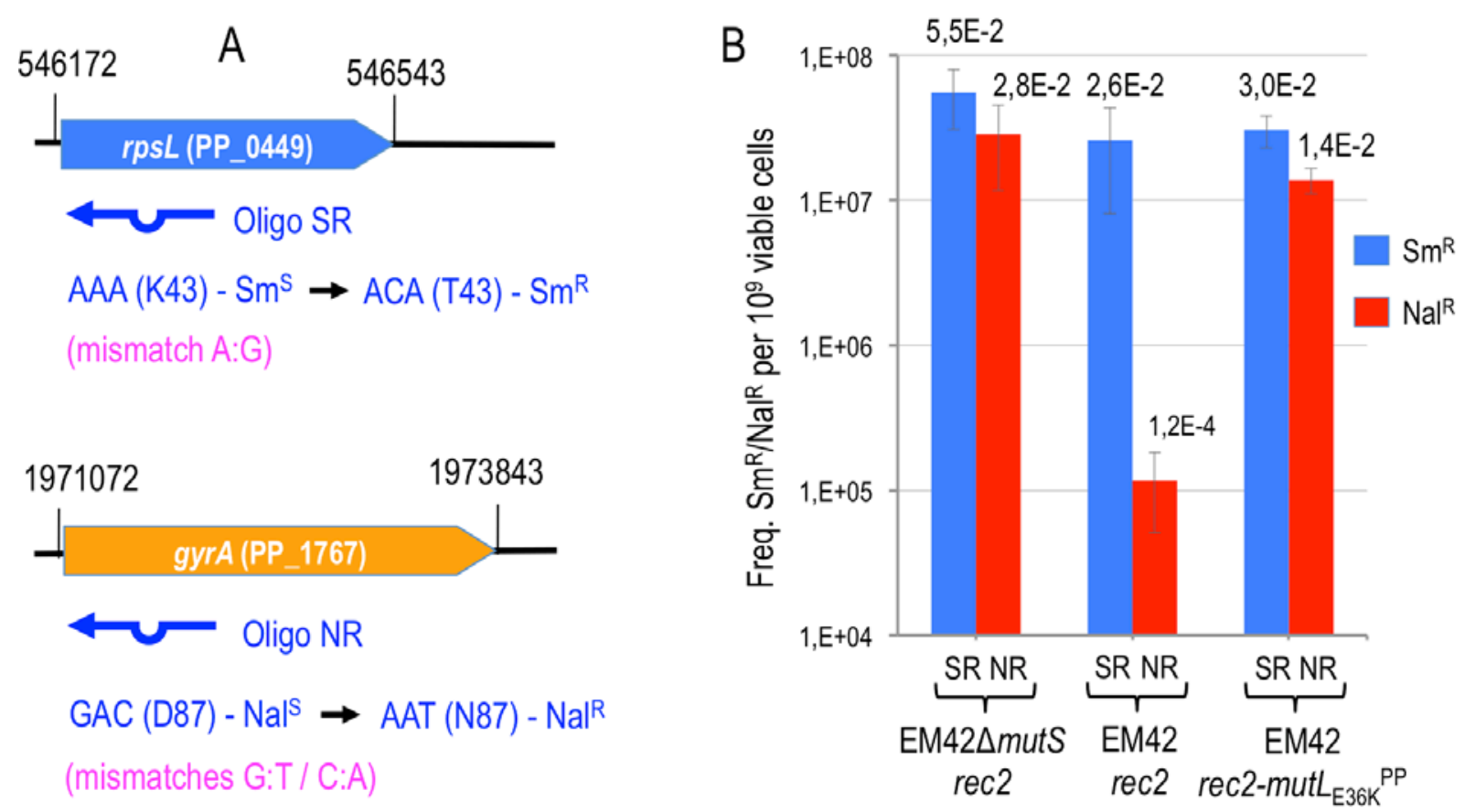

A. Reporter genes used to assess MMR system activity in P. putida EM42 are outlined. Chromosomal coordinates and locus tag are shown. Recombineering oligonucleotides are sketched below each gene, featuring the mutation introduced, the mismatch between chromosomal and synthetic sequences and also the resulting phenotype. B. Oligos SR (A:G mismatch, low MMR sensitivity, confers SmR) and NR (G:T and C:A mismatches, high MMR sensitivity, confer NalR) were used for recombineering in $P$. putida strains $\Delta$ mutS/pSEVA2514-rec2, EM42/pSEVA2514-rec2 and EM42/pSEVA2514-rec2-mutL ${ }_{E 36 K}{ }^{\mathrm{PP}}$. Cultures of each strain were subjected to recombineering with SR and NR oligonucleotides separately as explained in Experimental Procedures section. Dilutions of each experiment were plated on LB and LB-Sm (oligo SR) or LB-Nal (oligo NR) and colonies counted after $18 \mathrm{~h}$ at $30{ }^{\circ} \mathrm{C}$. Column values represent mean recombineering frequencies (mutants per $10^{9}$ viable cells) of two independent experiments with the standard deviation. Absolute frequencies (mutants per viable cell) are also shown above each column. 
Figure 3. Hierarchy of $P$. putida MMR system.
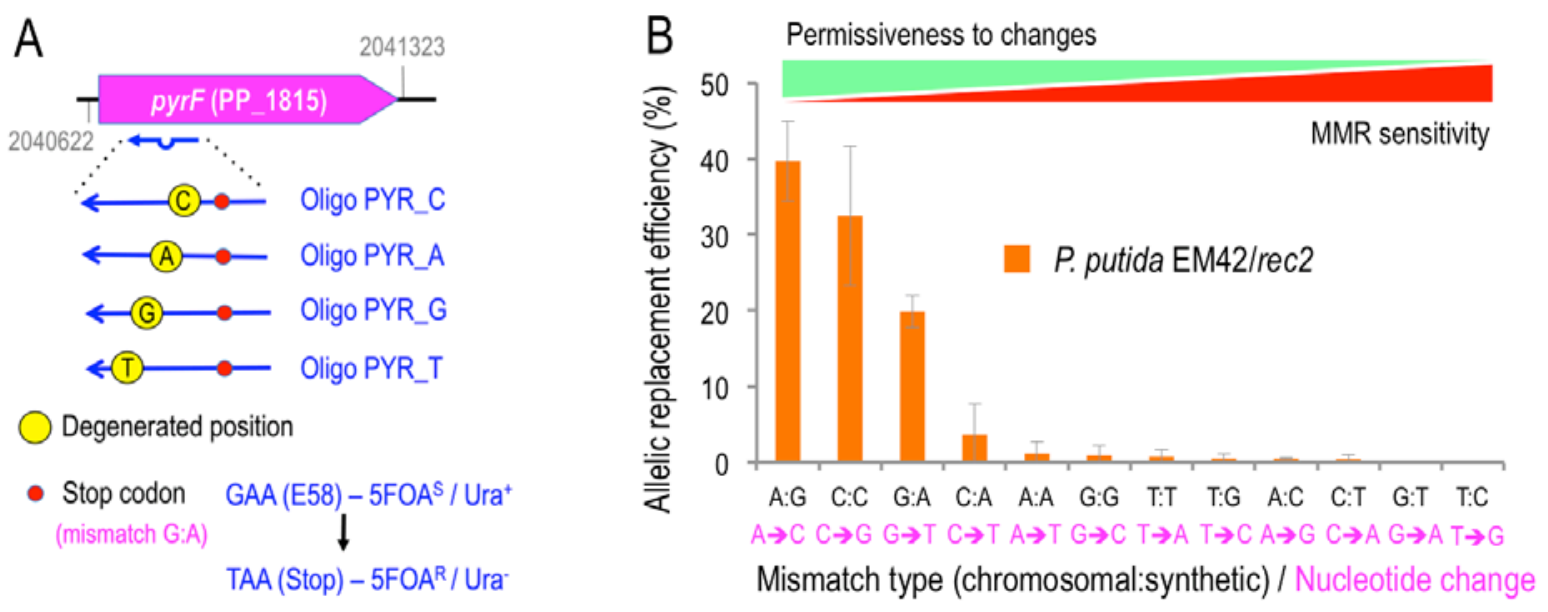

A. The pyrF reporter gene used to assess MMR hierarchy in P. putida EM42 is outlined. Locus tag and chromosomal coordinates are shown. The four PYR_X oligos introduce the same Stop codon (red dot), thus rendering a pyrF- strain which is uracil auxotroph and 5FOA resistant, but bear a different degenerated position each (yellow dot, the genomic nucleotide that pairs with oligonucleotide sequence is depicted inside), generating three mismatches per oligonucleotide. Pictures are not drawn to scale $\mathbf{B}$. P. putida EM42/pSEVA2514-rec2 (WT strain- wild-type MMR system) was subjected to recombineering with an equimolar mixture of oligos PYR_C, PYR_A, PYR_G and PYR_T. After selection of minimal media plus Ura/5FOA, 500 pyrF- colonies were streaked in the same media and the streaks resuspended in water, then pelleted and the whole genomic content extracted. pyrF gene was PCR amplified from the gDNA and sequenced by Illumina deep sequencing. Sequences were analysed to verify the presence of single mutations on the four degenerated positions targeted by PYR oligonucleotides. The relative frequencies of incorporated mutations were plotted and labelled with the original mismatch and the base change originated. The values are the mean of two independent experiments, bars representing standard deviations 
Figure 4. Effect of MMR inactivation on mismatch repair bias.
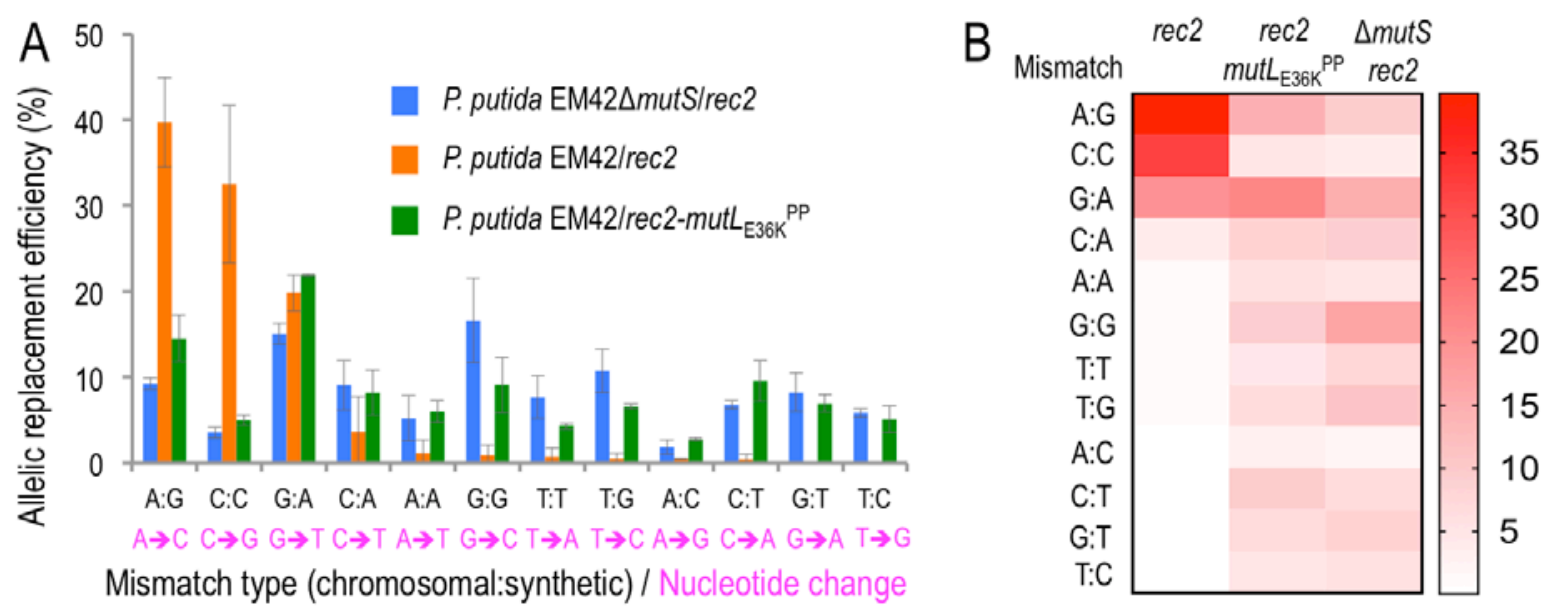

A. The same experiment as shown in Fig. 3 was performed using EM42AmutS/pSEVA2514-rec2 ( $\Delta$ mutS strain with an inactive MMR system) and EM42/pSEVA2514-rec2-mutL E36K ${ }^{\text {PP }}$ (MMR system transiently inhibited) strains and the results were compared with the wild-type scenario to study differences in the mutation bias under constitutive or transient impairment of MMR system, respectively. B. Heatmap of allelic replacement frequencies of the three strains under study. Detailed information of allelic replacement frequencies of every mismatch is shown in Supplementary Fig. S3. 
Figure 5. Mutation rates of $P$. putida EM42-derived strains.

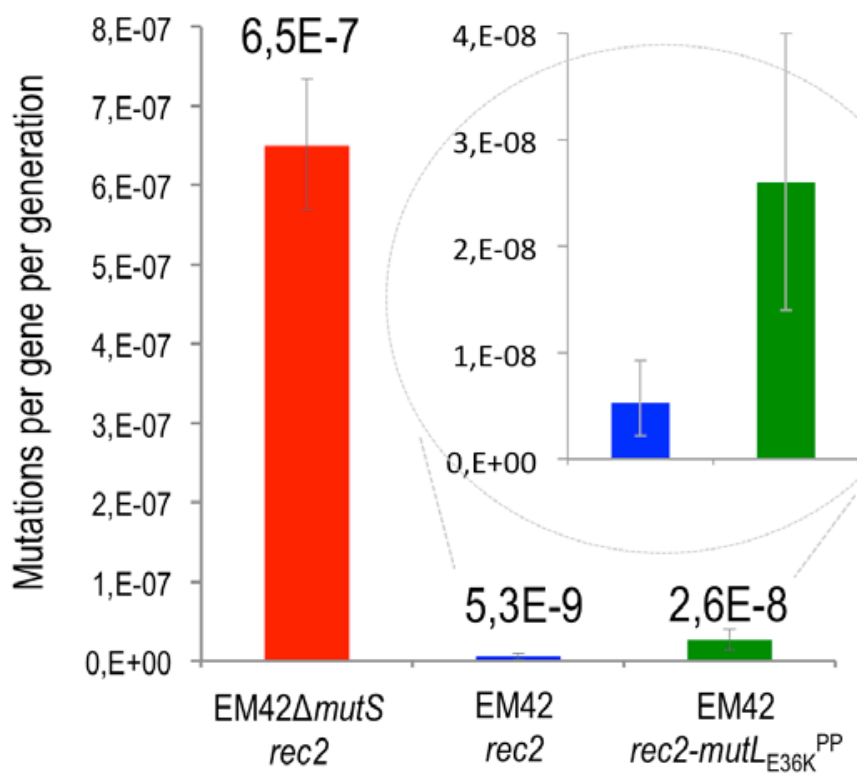

A rifampicin resistance fluctuation assay was used to estimate the mutation rates of EM42AmutS/pSEVA2514-rec2, EM42/pSEVA2514-rec2 and EM42/pSEVA2514-rec2-mutL E36K $^{\text {PP }}$ as described in Experimental Procedures. Fifteen independent replicas were performed and results analyzed with the FALCOR web tool by the MMS-Maximum Likelihood Estimator Method. FALCOR averages estimating mutations per gen per generation are depicted above the columns. Error bars account for the 95\% Confidence Intervals difference. A zoom-up has been included to better visualize the right part of the figure. 